\title{
Intranasal Administration of Oxytocin Attenuates Stress Responses Following Chronic Complicated Stress in Rats
}

\author{
Yu Yang, ${ }^{1}$ Haijie Yu, ${ }^{2}$ Reji Babygirija, ${ }^{3}$ Bei Shi, ${ }^{1}$ Weinan Sun, ${ }^{1}$ Xiaojiao Zheng, ${ }^{1}$ and Jun Zheng ${ }^{1 *}$ \\ ${ }^{1}$ Department of Physiology, School of Life Science, China Medical University, Shenyang; Liaoning Province, China; ${ }^{2}$ Department of Cardiology, \\ First Affiliated Hospital, China Medical University, Shenyang, Liaoning Province, China; and ${ }^{3}$ Department of Surgery, Medical College of \\ Wisconsin and Zablocki VA Medical Center, Milwaukee, WI, USA
}

\section{Background/Aims}

Gastrointestinal (GI) symptoms may develop when we fail to adapt to various stressors of our daily life. Central oxytocin (OXT) can counteract the biological actions of corticotropin-releasing factor (CRF), and in turn attenuates stress responses. Administration (intracerebroventricular) of OXT significantly antagonized the inhibitory effects of chronic complicated stress (CCS) on GI dysmotility in rats. However, intracerebroventricular administration is an invasive pathway. Intranasal administration can rapidly deliver peptides to the brain avoiding stress response. The effects of intranasal OXT on hypothalamus-pituitary-adrenal axis and GI motility in CCS conditions have not been investigated.

\section{Methods}

A CCS rat model was set up, OXT 5, 10, or $20 \mu \mathrm{g}$ were intranasal administered, 30 minutes prior to stress loading. Central CRF and OXT expression levels were analyzed, serum corticosterone and OXT concentrations were measured, and gastric and colonic motor functions were evaluated by gastric emptying, fecal pellet output, and motility recording system.

\section{Results}

Rats in CCS condition showed significantly increased CRF expression and corticosterone concentration, which resulted in delayed gastric emptying and increased fecal pellet output, attenuated gastric motility and enhanced colonic motility were also recorded. OXT $10 \mu \mathrm{g}$ or $20 \mu \mathrm{g}$ significantly reduced CRF mRNA expression and the corticosterone concentration, OXT $20 \mu \mathrm{g}$ also helped to restore Gl motor dysfunction induced by CCS.

\section{Conclusion}

Intranasal administration of OXT has an anxiolytic effect and attenuates the hypothalamus-pituitary-adrenal axis in response to CCS, and gave effects which helped to restore GI dysmotility, and might be a new approach for the treatment of stress-induced GI motility disorders.

(J Neurogastroenterol Motil 2019;25:611-622)

Key Words

Administration, intranasal; Corticosterone; Gastrointestinal motility; Oxytocin; Stress disorders

Received: March 28, 2019 Revised: June 4, 2019 Accepted: July 20, 2019

(a) This is an Open Access article distributed under the terms of the Creative Commons Attribution Non-Commercial License (http://creativecommons. org/licenses/by-nc/4.0) which permits unrestricted non-commercial use, distribution, and reproduction in any medium, provided the original work is properly cited.

${ }^{*}$ Correspondence: Jun Zheng, MD, PhD

Department of Physiology, School of Life Science, China Medical University, No.77 Puhe Road, North New Area, Shenyang, Liaoning Province 110122, China

Tel: +86-24-31939077, Fax: +86-24-31939077,E-mail: zheng_cmu@163.com

Yu Yang and Haijie Yu contributed equally to this study. 


\section{Introduction}

Functional gastrointestinal (GI) disorders, such as functional dyspepsia (FD) and irritable bowel syndrome (IBS), are common pathologies of the gut. The pathogeneses of FD and IBS are highly associated with stress in humans. GI dysmotility might develop when we fail to adapt to the accumulation of continuous or repeated stress in some individuals. ${ }^{1,2}$

Corticotropin-releasing factor (CRF) in the brain plays a significant role in the central nervous system mediation of stress-induced GI dysmotility. ${ }^{3-5}$ Acute restraint stress (ARS) delays gastric emptying (GE) via central CRF type $2\left(\mathrm{CRF}_{2}\right)$ receptors and sympathetic pathways. ${ }^{6}$ In contrast, accelerated colonic transit induced by $\mathrm{ARS}$ is mediated via central CRF type $1\left(\mathrm{CRF}_{1}\right)$ receptors and parasympathetic pathways in rats. ${ }^{7}$ Delayed GE and accelerated colonic transit were also observed in a chronic complicated stress (CCS) rat model, when rats received different types of stressors for 7-9 days, with elevated CRF mRNA expression in the paraventricular nucleus (PVN) of the hypothalamus, resulting in activation of the hypothalamus-pituitary-adrenal (HPA) axis. ${ }^{8,9}$

Until now, many peripheral selective $\mathrm{CRF}$ receptor antagonists have been developed, and could block the enhanced colonic motor function induced by stress, however, the efficiency of the antagonists is still controversial, the effects need to be further investigated. ${ }^{10-12}$

Further, a method of selective CRF deletion in the colon could prevent stress-related stimulation of colonic motor function in rats was also reported recently. ${ }^{13}$ However, the knockdown procedure is an invasive pathway, and its side-effects should require detailed investigation.

Oxytocin (OXT) is a cyclic nonapeptide hormone synthesized in the PVN and supraoptic nucleus of the hypothalamus. Besides its well-known physiological functions such as lactation and induction of labor, studies also suggest that central OXT has an anxiolytic effect and attenuates the HPA axis in response to stress. ${ }^{14,15}$ And recently, OXT application via the intranasal administration route has been shown to exert reduction of anxiety and can enhance social affiliation in rats, ${ }^{16}$ intranasal OXT is also a likely candidate for treatment of patients with post-traumatic stress disorder (PTSD). ${ }^{17,18}$ Intranasal administration method allows peptides to rapidly penetrate into the brain, bypassing the blood brain barrier to effectively interact with their receptors in multiple brain regions, without resorting to invasive administration procedures. ${ }^{19}$ Our previous study also showed that central administration of OXT (intracerebroventricular $[\mathrm{ICV}]$ ) significantly antagonized the inhibitory effects of acute stress and CCS on GI dysmotility, and there was a significant negative correlation observed between CRF and OXT expression in the PVN of the hypothalamus in rodents. ${ }^{8,20}$ However, ICV administration is an invasive pathway, which will produce stress response, and may affect the results.

Intranasal infusion represents a non-invasive approach for the rapid delivery of OXT to the brain that can avoid stress response. However, the effect of intranasal administration of OXT on HPA axis and GI motility have not been investigated.

Therefore, the present study sought to evaluate in CCS rats model, whether intranasal OXT have effects to attenuate the HPA axis activity in response to stress, also effects to help restore GI dysmotility. Our work will contribute to further studies on stressinduced abnormal GI motility and will help to seek a new approach (intranasal administration) for treatment of functional GI disorders.

\section{Materials and Methods}

\section{Animals}

Adult male Sprague-Dawley rats (laboratory animal center of China Medical University, Shenyang, China), weighing 260-300 $\mathrm{g}$, were housed in individual cages under conditions of controlled temperature $\left(22-24^{\circ} \mathrm{C}\right)$ and illumination (12-hour:12-hour lightdark cycle starting at 6 ante meridiem $[\mathrm{AM}]$ ) for at least 7 days before the experiment. Rats were fed with commercial pelleted feed from Xietong Organism Institute (Nanjing, China) and water ad libitum.

All experiments were started at 9 AM each day. Animal procedures were reviewed and approved by the Animal Care and Use Committee of China Medical University and conducted according to the guidelines of the laboratory animal ethical standards of China Medical University. The approval reference number is SCXK (Liao) 2016-0009.

\section{Chronic Complicated Stress Loading}

For CCS loading, the rats $(n=6-8)$ received different types of stressors for 7 consecutive days, as previously reported. ${ }^{8}$ The stress paradigms used were fasten restraint stress (FRS), force swimming stress (FSS), cold restraint stress (CRS), and water avoidance stress (WAS).

The specific conditions for each type of stress are as follows:

(1) FRS: rats were placed on a wooden plate with their trunks wrapped in a confining harness for 90 minutes. For the control group $(\mathrm{n}=6-8)$, the rats were housed in original individual 
cages for 90 minutes, but limited access to food and water.

(2) FSS: rats were placed individually in a plastic tank $(52 \times$ $37 \times 20 \mathrm{~cm}$ ) filled with room temperature $(\mathrm{RT})$ water to the depth of $15 \mathrm{~cm}$ for 20 minutes. The depth of the water forced the animal to swim or float without hindlimbs touching the bottom of the tank. Control rats were placed individually in a waterless container tank for 20 minutes.

(3) CRS: rats were kept restrained at $4{ }^{\circ} \mathrm{C}$ for 45 minutes. Control rats were kept at RT for 45 minutes.

(4) WAS: rats were placed on a platform $(3 \times 6 \mathrm{~cm})$ in the middle of a plastic container $(50 \times 30 \times 20 \mathrm{~cm})$ filled with RT water to $1 \mathrm{~cm}$ below the height of the platform for 60 minutes. Control rats were placed on the same platform in a waterless container for 60 minutes.

Rats were exposed to different stressors each day for 7 days:

Day 1: FRS (90 minutes, AM), FSS (20 minutes, post meridiem [PM]); Day 2: CRS (45 minutes, AM); Day 3: FRS (90 minutes, AM), WAS (60 minutes); Day 4: CRS (45 minutes, AM); Day 5: FRS (90 minutes, AM), FSS (20 minutes, PM); Day 6: CRS (45 minutes, AM); and Day 7: FRS (90 minutes, AM).

\section{Intranasal Administration of Oxytocin}

Rats received intranasal administration once a day, 30 minutes before everyday stress loading. CCS rats were divided into 4 groups ( $\mathrm{n}=6-8)$, infused with saline as control, synthetic 5, 10, or $20 \mu \mathrm{g}$ OXT (Sigma-Aldrich, St. Louis, MO, USA) freshly dissolved in $20 \mu \mathrm{L}$ water, and $10 \mu \mathrm{L}$ was infused into each nare with pipetteman and disposable plastic tip under light isoflurane anesthesia as previously described. ${ }^{21-23}$ Care was taken to avoid contact with the intranasal mucosa. Following intranasal administration, the head of the animal was held in a tilted back position for approximately 15 seconds to prevent loss of solution from the nares. For the nonstressed (NS) rats, they were also divided into 4 groups $(n=6-8)$, the rats were intranasal administrated with saline (as control), synthetic OXT 5, 10, and $20 \mu \mathrm{g}$, as mentioned above.

\section{Blood Collection and Hormone Assays}

The experimental rats were euthanized immediately by pentobarbital sodium $(200 \mathrm{mg} / \mathrm{kg}$ intraperitoneal injection; SigmaAldrich,), after stress loading. At the time of rats' death, trunk blood was collected immediately via a cardiac puncture, and then the samples were allowed to clot in tubes and centrifuged at $4{ }^{\circ} \mathrm{C}$ for 10 minutes at $3000 \mathrm{rpm}$ to separate out the serum. The serum fraction was stored at $-80^{\circ} \mathrm{C}$ for further analysis. Corticosterone con- centrations were measured by enzyme-linked immunosorbent assay (ELISA) using a corticosterone ELISA kit (Enzo Life Sciences, Plumoth meeting, PA, USA). OXT concentrations were measured by OXT ELISA kit (R\&D Systems Inc, Minneapolis, MN, USA). All procedures were carried out according to the manufacturer's instructions, as previously reported. ${ }^{24,25}$

\section{Quantitative Real-time Polymerase Chain Reaction}

The rat brain tissue micropunching technique was applied for acquiring hypothalamus tissue samples from specific regions with micro-punchers. Briefly, after stress loading, the experimental rats were euthanized and the brains were removed immediately and cut into $450 \mu \mathrm{m}$ coronal sections. Punches were collected from the left and right PVN (1.8 mm caudal to bregma; $0.4 \mathrm{~mm}$ lateral to midline; $7.6 \mathrm{~mm}$ ventral to the brain surface), as previously reported. ${ }^{8,24}$ All coordinates were based on the rat brain atlas and hypothalamic images of previously reported. ${ }^{26}$

Samples were stored at $-80^{\circ} \mathrm{C}$ until use. Total RNA was extracted from the brain tissues using Trizol (Invitrogen, Carlsbad, CA, USA), and trace DNA contamination was removed by DNase digestion (Promega, Madison, WI, USA). Complementary DNA was synthesized from $3 \mu \mathrm{g}$ total RNA by use of Superscript III reverse transcriptase (Invitrogen).

The following primers were designed to amplify rat CRF: sense primer 5'-CCAGGGCAGAGCAGTTAGCT-3', antisense primer 5'-CAAGCGCAACATTTCATTTCC-3'. The following were designed to amplify rat OXT: sense primer 5'-GAACACCAACGCCATGGCCTGCCC-3', antisense primer 5'-TCGGTGCGGCAGCCATCCGGGCTA-3'. For an internal control, the following were designed to amplify rat $\beta$-actin: sense primer 5'-TGGCACCACACCTTCTACAATGAG-3', antisense primer 5'-GGGTCATCTTTTCACGGTTGG-3', as previously reported. $^{8,24}$

Quantitative polymerase chain reaction (PCR) was performed by using SYBR Premix Ex Taq (TaKaRa Biotech, Dalian, China). Amplification reactions were performed using ABI 7500 Real-time PCR instrument (Applied Biosystems, San Mateo, CA, USA). Initial template denaturation was performed for 30 seconds at $95^{\circ} \mathrm{C}$. The cycle profiles were programmed as follows: 5 seconds at $95^{\circ} \mathrm{C}$ (denaturation), 20 seconds at $60^{\circ} \mathrm{C}$ (annealing), and 15 seconds at $72^{\circ} \mathrm{C}$ (extension). Forty-five cycles of the profile were run, and the final cooling step was continued for 30 seconds at $40^{\circ} \mathrm{C}$. Quantitative measurement of each messenger RNA (mRNA) sample was achieved by establishing a linear amplification curve from serial dilutions of each plasmid containing the amplicon sequence. Am- 
plicon size and specificity were confirmed by melting curve analysis. The relative amount of each $\mathrm{mRNA}$ was normalized by the amount of $\beta$-actin mRNA, as previously reported. ${ }^{8,24}$

\section{Measurement of Solid Gastric Emptying}

Rats were fasted for 24 hours prior to the measurement of GE. Preweighed standard rodent pellets ( $1.6 \mathrm{~g}$ ) were given, the rats that did not consume $1.6 \mathrm{~g}$ of food within 10 minutes were excluded from the study. For the control group, rats after finishing the feeding were put back into their original cages for 90 minutes, but with limited access to food and water, then the rats were euthanized by pentobarbital sodium. For the stress group, immediately after finishing the feeding, the rats were subjected to restraint stress for 90 minutes. Then the experimental rats were euthanized as mentioned above. The stomach was surgically isolated and removed. The gastric contents were recovered from the stomach, dried, and weighed. Solid GE was calculated according to the following formula, as previously described: $:^{8,24}$

$\mathrm{GE}(\%)=[1-($ dried weight of food recovered from stomach/weight of food intake) $] \times 100$

\section{Measurement of Fecal Pellet Output}

Rats were exposed to restraint stress treatment for 90 minutes as mentioned above, and the FPO numbers were counted after stress loading as measure of colonic transit function. For the control group, the rats after finishing the feeding were back into their original cages for 90 minutes, and the expelled fecal pellets were also counted, as previously reported. ${ }^{24}$

\section{Monitoring of Gastric and Colonic Motility}

Rats were anesthetized with isoflurane (2\%). Strain gauge transducers were implanted on the antrum for recording gastric contractions, and distal colon for colonic contractions. All wires were tunneled subcutaneously to exit at the back of the rat's neck and protected by a protective jacket (Star Medical, Tokyo, Japan). The abdominal wall was closed and rats were housed individually with access to a standard diet and tap water. After 7 days, the rats completely recovered from the surgery, including body weight and daily food intake.

Rats on a fixed-feeding schedule (food administered 2-6 PM daily) were monitored from $8 \mathrm{AM}$ to $4 \mathrm{PM}$ for gastric and colonic motility, as previously reported. ${ }^{24,27}$ The wires from the transducers were connected to a recording system (Power Lab 8SP; AD Instruments, Colorado Springs, CO, USA). Gastric and colonic contractions were monitored before, during, and after restraint stress.
Quantification of gastric motility and colonic motility were studied by the calculating motility index (MI). MI was equivalent with the area under the curve of the motility recording. MI was calculated using a computer-assisted system (Power Lab; AD Instruments), as previously reported. ${ }^{24,27}$

\section{Statistical Methods}

Analysis was performed using the SPSS version 20.0 statistical software (IBM Corp, Armonk, NY, USA). Results were shown as mean \pm standard error. Statistical analyses were performed using a two-way classification ANOVA to determine the significant interaction between different stress groups and drug treatment. Statistical significance was considered with a Bonferroni-adjusted alpha level of $P<0.05$.

\section{Results}

\section{Effects of Intranasal Administration of Oxytocin on Central CRF and oxytocin mRNA Expression in Response to Chronic Complicated Stress}

In the NS groups, CRF mRNA expression in the PVN showed low level, intranasal administration of OXT $(5,10$, or 20 $\mu \mathrm{g}$, respectively, daily, before stress loading) did not change the $C R F$ mRNA expression significantly, compared to that of saline intranasal administrated NS rats (saline $10 \mu \mathrm{L}$ as a control, $\mathrm{n}=6$ ). In the CCS groups, CRF mRNA expression increased significantly compared to that of saline intranasal administrated NS rats $(n=6)$. Intranasal administration of OXT $5 \mu \mathrm{g}$ did not change the $C R F$ mRNA expression significantly, however, intranasal administration of OXT $10 \mu \mathrm{g}$ and $20 \mu \mathrm{g}$ significantly decreased the CRF mRNA expression compared to that of saline intranasal administrated CCS rats $(\mathrm{n}=6, P<0.05$, saline $10 \mu \mathrm{L}$ intranasal administrated as a control; Fig. 1A).

In the NS groups, oxytocin mRNA expression in the PVN showed very low level, intranasal administration of $\operatorname{OXT}(5,10$, or $20 \mu \mathrm{g}$, respectively, daily, before stress loading) did not change the oxytocin mRNA expression significantly, compared to that of saline intranasal administrated NS rats (saline $10 \mu \mathrm{L}$ as a control, $\mathrm{n}=$ 6). In the CCS groups, oxytocin mRNA expression did not change significantly compared to that of saline intranasal administrated NS rats $(n=6)$. Intranasal administration of OXT $5 \mu \mathrm{g}$ and $10 \mu \mathrm{g}$ did not change the oxytocin mRNA expression significantly, however, intranasal administration of OXT $20 \mu \mathrm{g}$ significantly increased the oxytocin mRNA expression compared to that of saline intranasal 
A

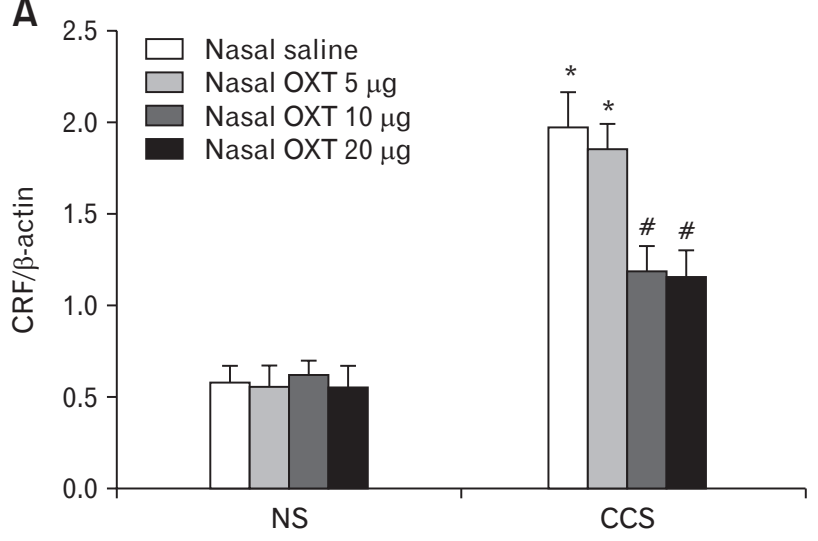

B

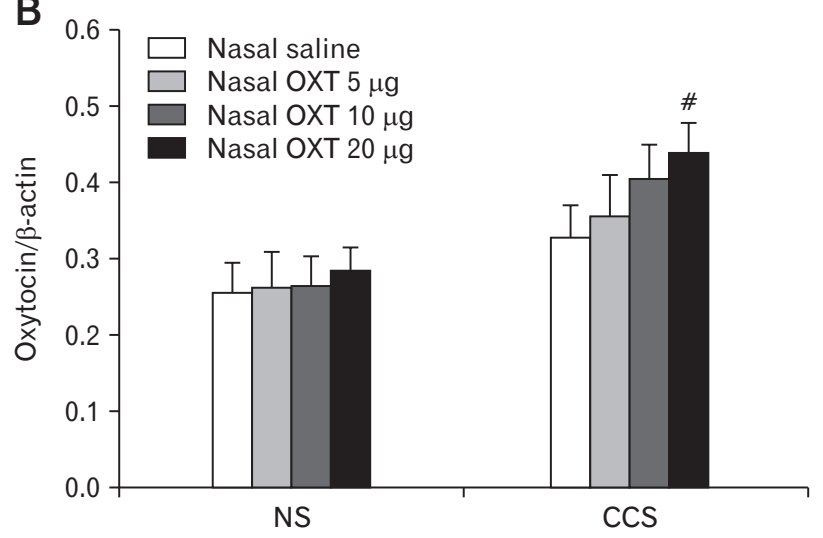

Figure 1. Effects of intranasal administration of oxytocin (OXT) on central corticotropin-releasing factor (CRF) (A) and oxytocin (B) mRNA expression in response to chronic complicated stress (CCS). (A) In the non-stressed (NS) groups, OXT had no effect on the CRF mRNA expression. In the CCS groups, $C R F$ mRNA expression increased significantly. However, OXT $10 \mu \mathrm{g}$ and $20 \mu \mathrm{g}$ significantly decreased the $C R F$ mRNA expression. (B) In the NS groups, OXT had no effect on the oxytocin mRNA expression. In the CCS groups, oxytocin mRNA expression did not significantly change in nasal saline as well as OXT $5 \mu \mathrm{g}$ and $10 \mu \mathrm{g}$. However, OXT $20 \mu \mathrm{g}$ significantly increased the oxytocin mRNA expression. The mRNA expression was standardized with the ratio of internal control of $\beta$-actin $\left(\mathrm{n}=6,{ }^{*} P<0.05\right.$ compared with NS nasal saline group, ${ }^{\#} \mathrm{P}<0.05$ compared with CCS nasal saline group).

administrated CCS rats $(\mathrm{n}=6, P<0.05$, saline $10 \mu \mathrm{L}$ intranasal administrated as a control; Fig. 1B).

\section{Effects of Intranasal Administration of Oxytocin on Serum Corticosterone and Oxytocin Levels in Response to Chronic Complicated Stress}

In the NS groups, serum corticosterone concentration showed low level. Intranasal administration of $\operatorname{OXT}(5,10$, or $20 \mu \mathrm{g}$, daily, before stress loading) did not change the serum corticosterone levels significantly $(65.9 \pm 7.4 \mathrm{ng} / \mathrm{mL}, 63.1 \pm 7.5 \mathrm{ng} / \mathrm{mL}$, and 61.2 $\pm 8.5 \mathrm{ng} / \mathrm{mL}$, respectively) compared to that of saline intranasal administrated rats $(65.5 \pm 6.9 \mathrm{ng} / \mathrm{mL}$, saline $10 \mu \mathrm{L}$ intranasal administrated as a control, $\mathrm{n}=6$ ). In the CCS groups, serum corticosterone concentration significantly increased to $145.6 \pm 12.2 \mathrm{ng} /$ $\mathrm{mL}$ compared to that of saline intranasal administrated NS rats (n $=6, P<0.025)$. Intranasal administration of OXT $5 \mu \mathrm{g}$ did not change the serum corticosterone level significantly $(139.9 \pm 13.2$ $\mathrm{ng} / \mathrm{mL}, \mathrm{n}=6$ ), however, intranasal administration of OXT $10 \mu \mathrm{g}$ and $20 \mu \mathrm{g}$ significantly decreased the corticosterone level to $97.1 \pm$ $8.2 \mathrm{ng} / \mathrm{mL}$ and $94.2 \pm 8.8 \mathrm{ng} / \mathrm{mL}$, respectively, compared to that of saline intranasal administrated CCS rats $(\mathrm{n}=6, P<0.05$, saline $10 \mu \mathrm{L}$ intranasal administrated as a control; Fig. 2A).

In the NS groups, serum OXT concentration showed very low level. Intranasal administration of $\operatorname{OXT}(5,10$, and $20 \mu \mathrm{g}$, daily, before stress loading) did not change the serum OXT levels significantly $(198 \pm 22 \mathrm{pg} / \mathrm{mL}, 213 \pm 17 \mathrm{pg} / \mathrm{mL}$, and $214 \pm 21$ $\mathrm{pg} / \mathrm{mL}$, respectively), compared to that of saline intranasal administrated rats $(200 \pm 18 \mathrm{ng} / \mathrm{mL}$, saline $10 \mu \mathrm{L}$ intranasal administrated as a control, $\mathrm{n}=6)$. In the CCS groups, serum OXT concentration did not change significantly $(259 \pm 20 \mathrm{pg} / \mathrm{mL})$ compared to that of saline intranasal administrated NS rats $(\mathrm{n}=6, P<0.025)$. Intranasal administration of OXT $5 \mu \mathrm{g}$ and $10 \mu \mathrm{g}$ did not change the serum OXT level significantly $(275 \pm 22 \mathrm{pg} / \mathrm{mL}$ and $308 \pm$ $27 \mathrm{pg} / \mathrm{mL}$, respectively, $\mathrm{n}=6$ ), however, intranasal administration of OXT $20 \mu \mathrm{g}$ significantly increased the OXT level to $351 \pm 24$ $\mathrm{pg} / \mathrm{mL}$, compared to that of saline intranasal administrated CCS rats (n $=6, P<0.05$, saline $10 \mu \mathrm{L}$ intranasal administrated as a control; Fig. 2B).

\section{Effects of Intranasal Administration of Oxytocin on Gastric Emptying and Fecal Pellet Output in Response to Chronic Complicated Stress}

In the NS groups, GE showed normal level. Intranasal administration of $\operatorname{OXT}(5,10$, and $20 \mu \mathrm{g}$, daily, before stress loading) did not change the GE significantly $(51.5 \pm 3.8 \%, 50.9 \pm$ $3.0 \%$, and $53.2 \pm 3.2 \%$, respectively) compared to that of saline intranasal administrated rats $(49.2 \pm 3.2 \%$, saline $10 \mu \mathrm{L}$ intranasal administrated as a control, $\mathrm{n}=6$ ). In the CCS groups, $\mathrm{GE}$ was significantly decreased to $23.1 \pm 2.8 \mathrm{ng} / \mathrm{mL}$ compared to that of saline intranasal administrated NS rats $(n=6, P<0.025)$. Intranasal administration of OXT $5 \mu \mathrm{g}$ and $10 \mu \mathrm{g}$ did not change the GE significantly $(25.1 \pm 2.3 \%$ and $29.1 \pm 2.9 \%$, respectively, $\mathrm{n}=$ 
6), however, intranasal administration of OXT $20 \mu \mathrm{g}$ significantly increased the GE to $43.1 \pm 3.2 \%$, compared to that of saline intranasal administrated CCS rats $(\mathrm{n}=6, P<0.025$, saline $10 \mu \mathrm{L}$ intranasal administrated as a control; Fig. 3A).

In the NS groups, FPO were $3.0 \pm 0.41$ (number/90 minutes). Intranasal administration of $\operatorname{OXT}(5,10$, and $20 \mu \mathrm{g}$, daily, before stress loading) did not change the FPO significantly (3.5 \pm $0.6,3.3 \pm 0.5$, and $3.6 \pm 0.6$ (number/90 minutes), respectively, $\mathrm{n}=6)$ compared to that of saline intranasal administrated rats (3.1 \pm 0.7 , saline $10 \mu \mathrm{L}$ intranasal administrated as a control, $\mathrm{n}=6$ ). In the CCS groups, FPO was significantly increased to $9.5 \pm 0.8$ (number/90 minutes, $\mathrm{n}=6$ ) compared to that of saline intranasal administrated NS rats $(\mathrm{n}=6, P<0.025)$. Intranasal administration of OXT $5 \mu \mathrm{g}$ and $10 \mu \mathrm{g}$ did not change the FPO significantly $(9.6 \pm 0.9$ and $7.3 \pm 0.6$, respectively, $\mathrm{n}=6)$, however, intranasal administration of OXT $20 \mu \mathrm{g}$ significantly decreased the FPO to
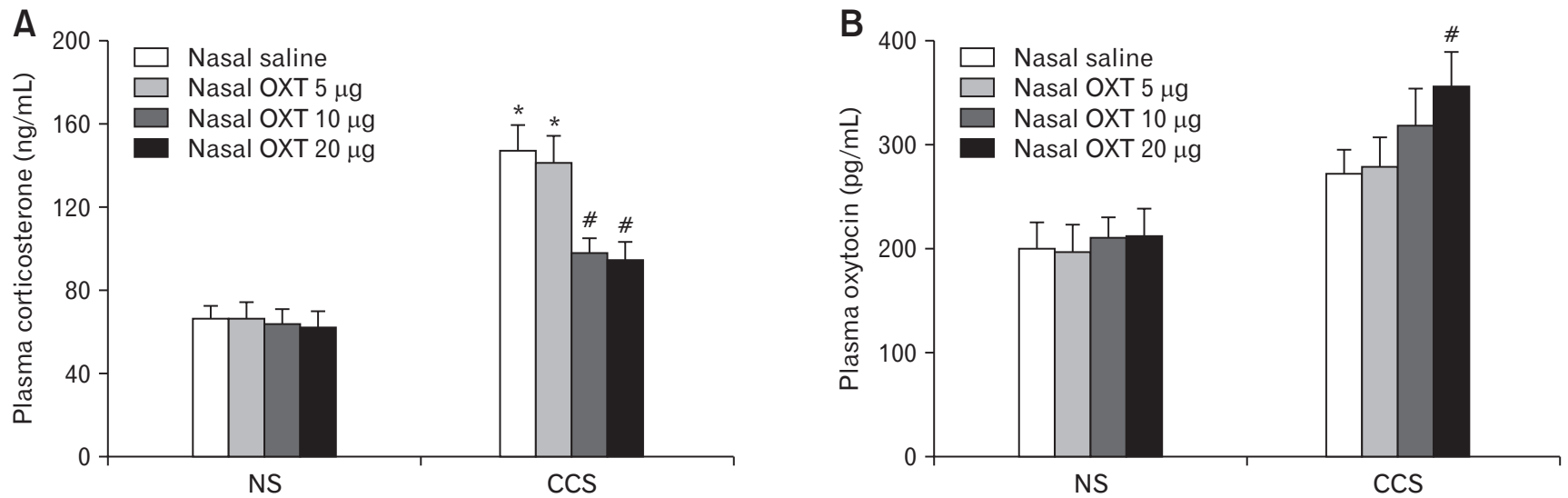

Figure 2. Effects of intranasal administration of oxytocin (OXT) on serum corticosterone (A) and OXT (B) levels in response to chronic complicated stress (CCS). (A) In the non-stressed (NS) groups, OXT had no effect on the serum corticosterone levels. In the CCS groups, corticosterone levels increased significantly (nasal saline). OXT $5 \mu \mathrm{g}$ did not change the corticosterone levels significantly, however, OXT $10 \mu \mathrm{g}$ and $20 \mu \mathrm{g}$ significantly decreased the corticosterone levels. (B) In the NS groups, OXT had no effect on the OXT level. In the CCS groups, OXT level did not significantly change in nasal saline as well as OXT $5 \mu \mathrm{g}$ and $10 \mu \mathrm{g}$. However, OXT $20 \mu \mathrm{g}$ significantly increased the OXT level $(\mathrm{n}=6$, *P $<$ 0.05 compared with NS nasal saline, ${ }^{*} \mathrm{P}<0.05$ compared with CCS nasal saline).
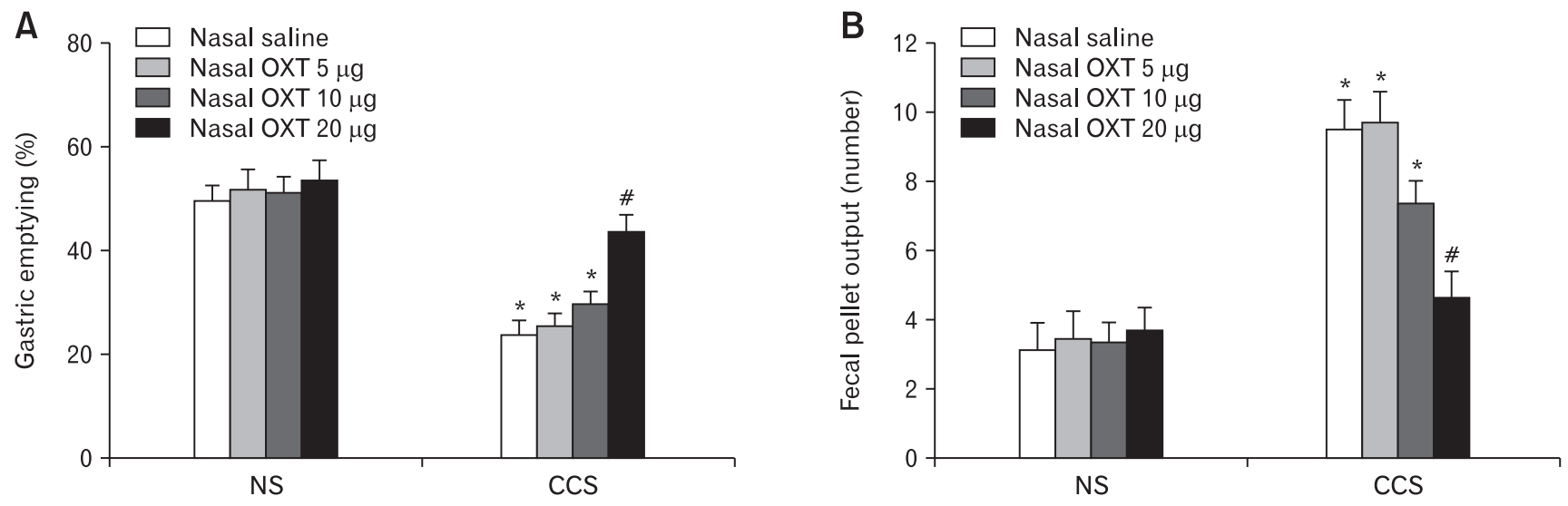

Figure 3. Effects of intranasal administration of oxytocin (OXT) on gastric emptying (GE; A) and fecal pellet output (FPO; B) in response to chronic complicated stress (CCS). (A) In the non-stressed (NS) groups, OXT had no effect on GE. In the CCS groups, GE was significantly delayed (nasal saline). OXT $5 \mu \mathrm{g}$ and $10 \mu \mathrm{g}$ did not change GE significantly, however, OXT $20 \mu \mathrm{g}$ significantly increased GE. (B) In the NS groups, OXT had no effect on the number of FPO. In the CCS groups, FPO significantly increased (nasal saline). OXT $5 \mu \mathrm{g}$ and $10 \mu \mathrm{g}$ did not change FPO significantly, however, OXT $20 \mu \mathrm{g}$ significantly decreased FPO (n $=6,{ }^{*} \mathrm{P}<0.05$ compared with NS nasal saline, ${ }^{\#} \mathrm{P}<0.05$ compared with CCS nasal saline). 
$5 \pm 0.5$ (number/90 minutes, $\mathrm{n}=6$ ), compared to that of saline intranasal administrated CCS rats $(\mathrm{n}=6, P<0.05$, saline $10 \mu \mathrm{L}$ intranasal administrated as a control; Fig. 3B).

\section{Effects of Intranasal Administration of Oxytocin on Gastric and Colonic Motility in Response to Chronic Complicated Stress}

\section{Motility recording}

In the NS groups, intranasal administration of $\operatorname{OXT}(5,10$, and $20 \mu \mathrm{g}$, daily, before stress loading) had no effects on the amplitude and frequency of gastric phase III-like contractions (data not shown), saline $10 \mu \mathrm{L}$ intranasal administrated as a control. In the CCS groups, restraint stress abolished gastric phase III-like contractions in the saline group (saline $10 \mu \mathrm{L}$ intranasal administered as a control). Intranasal administration of OXT ( $5 \mu \mathrm{g}$, daily, before stress loading) had no effects on the amplitude and frequency of gastric phase III-like contractions. However, gastric phase III-like contractions were restored partially at intranasal administration of OXT (10 $\mu \mathrm{g}$ and $20 \mu \mathrm{g}$, daily, before stress loading) groups (Fig. $4 \mathrm{~A})$.

In the NS groups, intranasal administration of $\operatorname{OXT}(5,10$, and $20 \mu \mathrm{g}$, daily, before stress loading) had no effects on the amplitude of distal colonic contraction (data not shown), saline $10 \mu \mathrm{L}$ intranasal administrated as a control. In the CCS groups, restraint stress enhanced the amplitude of distal colonic contraction in the saline group (saline $10 \mu \mathrm{L}$ intranasal administered as a control). Intranasal administration of OXT ( $5 \mu \mathrm{g}$, daily, before stress load- ing) had no effects on the amplitude of distal colonic contraction. However, distal colonic contraction was restored partially at intranasal administration of OXT (10 $\mu \mathrm{g}$ and $20 \mu \mathrm{g}$, daily, before stress loading) groups (Fig. 4B).

Each recording experiment was individually repeated at least three times and similar results were obtained (saline $10 \mu \mathrm{L}$ intranasal administered as a control, $n=4$; Fig. 4).

\section{Motility index changes}

In the NS groups, intranasal administration of $\operatorname{OXT}(5,10$, and $20 \mu \mathrm{g}$, daily, before stress loading) did not significantly alter the gastric MI change $(102 \pm 10 \%, 104 \pm 9 \%$, and $105 \pm 6 \%$, respectively) compared to that of saline intranasal administrated rats (103 $\pm 8 \%$, saline $10 \mu \mathrm{L}$ intranasal administrated as a control, $\mathrm{n}=$ 6; Fig. 5A). In the CCS groups, the attenuated gastric MI change in the saline group ( $48 \pm 5 \%$, saline $10 \mu \mathrm{L}$ intranasal administered as a control), was significantly enhanced by Intranasal administration of OXT (10 $\mu \mathrm{g}$ and $20 \mu \mathrm{g}$, daily, before stress loading, to 75 $\pm 4 \%$ and $83 \pm 7 \%$ ), however, intranasal administration of OXT (5 $\mu \mathrm{g}$, daily, before stress loading ) had no effects on the MI change (51 $\pm 5 \%)$, compared to that of saline intranasal administrated CCS rats ( $\mathrm{n}=6, P<0.05$, saline $10 \mu \mathrm{L}$ intranasal administrated as a control; Fig. 5A).

In the NS groups, intranasal administration of $\operatorname{OXT}(5,10$, and $20 \mu \mathrm{g}$, daily, before stress loading) did not significantly alter the colonic MI change $(100 \pm 10 \%, 102 \pm 8 \%$, and $97 \pm 11 \%$, respectively) compared to that of saline intranasal administrated rats $(99 \pm 10 \%$, saline $10 \mu \mathrm{L}$ intranasal administrated as a control,

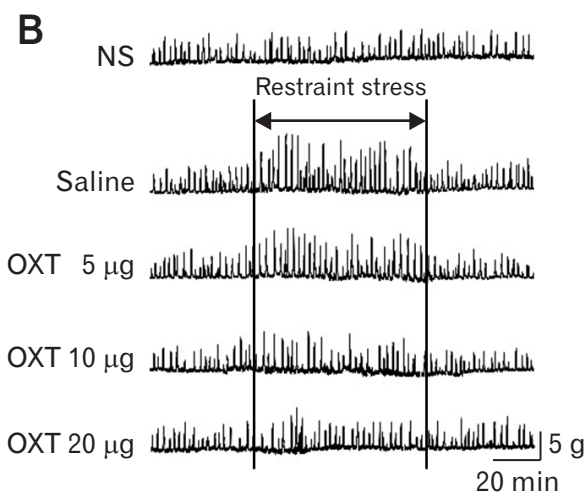

Figure 4. Effects of intranasal administration of oxytocin (OXT) on gastric (A) and colonic (B) motility in response to chronic complicated stress (CCS). (A) The gastric phase III-like contractions in the non-stressed (NS) groups. CCS abolished gastric phase III-like contractions in the nasal saline group (Saline). OXT $(5 \mu \mathrm{g})$ had no effect on gastric contractions. OXT (10 $\mu \mathrm{g})$ partially restored gastric contractions. OXT (20 $\mu \mathrm{g})$ further restored gastric phase III-like contractions. (B) The distal colonic contractions in the NS groups. CCS strongly enhanced the amplitude of colonic contractions in the nasal saline group (Saline). OXT (5 $\mu \mathrm{g})$ had no obvious effect on colonic contractions. OXT (10 $\mu \mathrm{g})$ partially attenuated colonic contractions. OXT $(20 \mu \mathrm{g})$ further attenuated colonic contractions. 

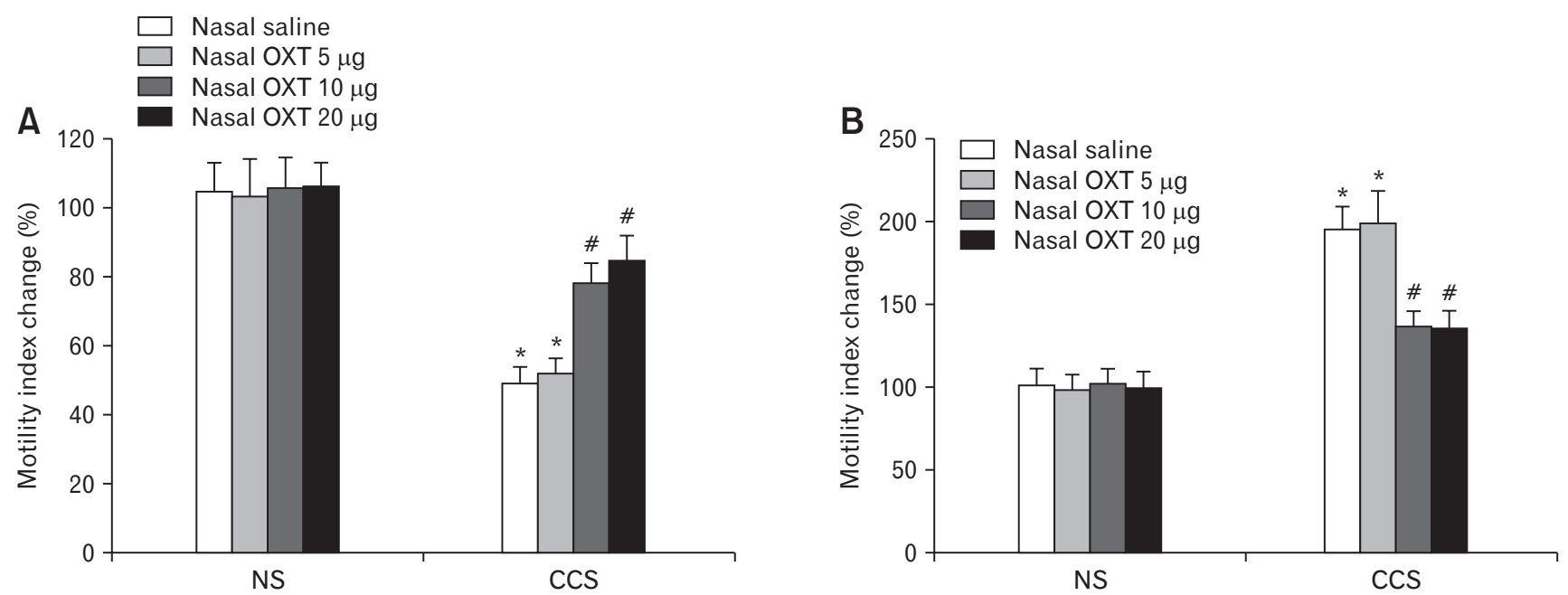

Figure 5. Effects of intranasal administration of oxytocin (OXT) on gastric (A) and colonic (B) motility index (MI) change in response to chronic complicated stress (CCS). (A) OXT did not significantly alter gastric MI change in the non-stressed (NS) groups. In the CCS groups, MI was significantly attenuated (nasal saline). OXT $5 \mu \mathrm{g}$ had no effect on the MI change, however, OXT $10 \mu \mathrm{g}$ and $20 \mu \mathrm{g}$ significantly enhanced MI change. (B) OXT did not significantly alter the colonic MI change in the NS groups. In the CCS groups, colonic MI change was significantly increased (nasal saline). OXT $5 \mu \mathrm{g}$ had no effect on MI change, however, OXT $10 \mu \mathrm{g}$ and $20 \mu \mathrm{g}$ significantly decreased MI change (n $=6$, * $\mathrm{P}<$ 0.05 compared with NS nasal saline, ${ }^{\#} P<0.05$ compared with CCS nasal saline).

$\mathrm{n}=6$; Fig. 5B). In the CCS groups, the enhanced colonic MI change in the saline group $(193 \pm 14 \%$, saline $10 \mu \mathrm{L}$ intranasal administered as a control), was significantly attenuated by intranasal administration of OXT ( $10 \mu \mathrm{g}$ and $20 \mu \mathrm{g}$, daily, before stress loading, to $138 \pm 12 \%$ and $133 \pm 10 \%)$, however, intranasal administration of OXT ( $5 \mu \mathrm{g}$, daily, before stress loading $)$ had no effects on the colonic MI change (197 $\pm 14 \%)$, compared to that of saline intranasal administrated CCS rats $(\mathrm{n}=6, P<0.05$, saline $10 \mu \mathrm{L}$ intranasal administrated as a control; Fig. 5B).

\section{Discussion}

Restraint stress has been used frequently as a physical and psychogenic stress model in rodents. In contrast to ARS, chronic repeated restraint stress produce habituation or diminution of behavioral responses. Our previous studies shown that in repeated restraint stress conditions, the elevated central CRF expression and serum corticosterone level, caused by ARS, was attenuated on day 5 and 7 of consecutive stress loading. ARS-induced GI dysmotility, such as delayed GE, impaired gastric phase III-like contractions and accelerated FPO, gradually returned to normal levels. ${ }^{24,27}$

In the present study, we set up a CCS model, and found that attenuated gastric motility (including delayed GE and impaired gastric phase III-like contractions) and enhanced colonic motility (including increased FPO and accelerated colonic transit) were also observed when rats received different types of stressors for 7-9 days, with highly elevated $C R F$ mRNA expression in the PVN of the hypothalamus, resulting in activation of the HPA axis. These results are consistent with previous studies, ${ }^{8,9}$ and may support the conclusion that $\mathrm{CRF}$ is one of the major mediators involved in inducing changes in the body in response to stress, CRF in lower amounts may be critical in focusing the attention to counter the potential damage, however, its massive release during persistent stress contributes to producing deleterious changes. ${ }^{12}$

A previous study reported that peripheral injection of CRF induces accelerated colonic motor activity in rats, ${ }^{10}$ and it seems that peripheral administration of CRF elicits similar alterations of gut motor function than central administration. ${ }^{28}$ However, our recent study also found that intracisternal injection of CRF still had an inhibitory effect on GE in chronically stressed rats, even though chronic stress showed no further inhibitory effects on GE. This suggested that the inhibitory pathway in response to central CRF is not altered following chronic stress. ${ }^{27} \mathrm{CRF}$ delays $\mathrm{GE}$ via central $\mathrm{CRF}_{2}$ receptors and sympathetic pathways. ${ }^{6}$ However, accelerated colon transit induced by ARS is mediated via central $\mathrm{CRF}_{1}$ receptors and parasympathetic pathways in rats. ${ }^{7}$ Until now there are no data on how central CRF signaling interacts with peripheral CRF. However, there is evidence that peripheral CRF acts on myenteric neurons that determine the activity of gastric and colonic functions during stress, ${ }^{29-31}$ and further studies are needed to elucidate these 
issues.

Gastric motility consists of 2 motor patterns: postprandial contractions and interdigestive contractions. Postprandial contractions mediate GE, whereas in the interdigestive state, showing a remarkable motor pattern, known as the migrating motor complex (MMC). MMC consists of phase I (period of motor quiescence), phase II (period of irregular low-amplitude), and phase III (period of regular high amplitude) contractions. MMC plays an important role in mediating mechanical and chemical cleansing of the empty stomach in preparation for the next meal. When gastric phase III is impaired, the gastric content may stay for a longer period. Impaired gastric phase III may cause retention of the gastric contents, bacterial overgrowth, and delayed GE, resulting in various symptoms. ${ }^{32}$ The characteristic feature of gastric MMC is different among species. In humans and dogs, MMC is usually observed every 90-120 minutes in the interdigestive state. In contrast, in rats, the MMC cycle is short (less than 20 minutes) and not as regular as that of humans and dogs. ${ }^{32}$ It is rather difficult to distinguish the 3 phases, especially phase III-like contractions. Furthermore, sometimes phase III-like contractions are not clearly observed even after 24hour fasting in rats. 32,33

Previous studies have shown that a fixed-feeding schedule (food administered 2-6 PM daily) could facilitate the occurrence of regular and potent phase III-like contractions in conscious rats. Fixedfed rats could gain their body weight and daily growth of their body weight is almost the same as that of free-fed rats. ${ }^{33}$ Our previous and present study also did not find that fixed-feeding schedule could affect the results in the chronic stress rat model. ${ }^{8,24,27}$

OXT plays an important role in regulating social behavior and positive social interactions in nonhuman mammals. ${ }^{34}$ In humans, intranasal administration of OXT was shown to have a substantial increase in trusting behavior, ${ }^{35}$ even enhance social processing in people with schizophrenia, ${ }^{36}$ and has the potential to enhance social reinforcement learning in autism spectrum disorders. ${ }^{37}$ It has been shown that OXT attenuates stress-induced responses and that released OXT may buffer an organism from the effects of stress via attenuating activity of HPA axis. ${ }^{14,15}$ The stress induced patterns of local release of OXT are dependent on various factors, such as sex, type of stressor, and the genetically determined stress susceptibility. ${ }^{38,39}$ Our previous study showed that restraint stress, a moderate degree of stimulus, significantly increased oxytocin mRNA expression in the PVN of the hypothalamus. In CRS loading for 5-7 days, oxytocin mRNA expression was significantly highly elevated, central OXT plays an important role in mediating the adaptation mechanism against chronic stress. ${ }^{8,40}$ However, in CCS conditions, no adaptation developed, oxytocin mRNA expression in the PVN of the hypothalamus has no significant elevation. ${ }^{8}$ As mentioned above, hypothalamic OXT might only react to moderate or slight degrees of chronic stress, while for the higher intensity stress condition, OXT system is exhausted, and central OXT failed to play an important role to produce an adaptation. Thus, external administration of OXT was expected in the termination of the stress response of $\mathrm{CCS}$ condition.

Our previous study showed that ICV-injection of OXT significantly attenuated the increase of $C R F \mathrm{mRNA}$ expression at the PVN induced by ARS in rats. ${ }^{8}$ However, ICV-administration of OXT, which was ineffective on GE in normal conditions, significantly improved delayed GE induced by ARS in rats, ${ }^{8}$ suggesting that central OXT may play a predominant role in regulating stressinduced GI dysmotility, but not in NS conditions. Our present study also showed that intranasal administration of OXT significantly improved delayed GE and impaired gastric motility, and also significantly attenuated the accelerated FPO and enhanced distal colonic motility induced by CCS in rats. Furthermore, intranasal administration of OXT was ineffective on CRF expression and corticosterone levels in NS conditions, as well as GI motor functions. These results support the previous study that central OXT is only effective under the stressful conditions. ${ }^{8,41}$

However, the majority of neuronal responses to OXT are excitatory, the inhibitory effect of OXT may not directly act on CRF neurons. ${ }^{42}$ Recent studies showed that central OXT inhibits stressinduced $C R F$ mRNA expression may be via gamma-aminobutyric acid (GABA)-A receptors in the PVN. ${ }^{43}$ GABA is the major inhibitory amino acid transmitter of the mammalian central nervous system. GABA exerts its effects through GABA-A and GABA-B receptors. GABA-projecting neurons into the PVN are known to inhibit CRF expression via GABA-A receptors. ${ }^{44}$ Released corticosterone in response to acute stress inhibits CRF release via a feedback mechanism, which is mediated via GABA-A receptors in the PVN. ${ }^{45}$ OXT enhances GABAergic transmission through a rapid and reversible increase of the frequency of the inhibitory postsynaptic currents. ${ }^{46}$ Thus, whether the inhibitory mechanism of intranasal administration of OXT on CRF mRNA expression is mediated via GABA-A receptors in the PVN in chronic stress conditions, is needed in a future study.

Besides the role in the central system, OXT may exert its effect on GI system also through the peripheral system. ${ }^{40,47}$ OXT receptors are also expressed on the smooth muscle cells, enteric neurons, and intestinal epithelium of the GI tract in rats. ${ }^{48.49}$ Our present study found that in CCS conditions, intranasal administration of 
OXT $10 \mu \mathrm{g}$ or $20 \mu \mathrm{g}$ significantly increased the serum OXT concentration, which is not controversial with a previous study. ${ }^{22}$ Highly elevated serum OXT concentration may benefit for the recovery of GI motor function. ${ }^{40,41}$ However, the peripheral mechanism of OXT exerting its effect on GI system in chronic stress conditions remains to be determined, and further investigation is needed in future studies.

The methodology of intranasal administration OXT has been developed, and OXT application has been shown to exert reduction of anxiety and can enhancing social affiliation in rats by recent studies. $^{22,23}$ However, the conscious rat was restrained to the holding position when OXT was administrated, and needed 1 week of habituation in the holding position, which is in a mild chronic restraint stress condition, will activate HPA axis, and may produce adaptation, which may affect the results of our current study. To avoid this, when giving OXT administration, rats were under light isoflurane anesthesia as the methodology previously described. ${ }^{21}$ Further, the dose of OXT $20 \mu \mathrm{g}$ for intranasal administration in rats is a relative higher dose, compared to humans, usually up to $48 \mu \mathrm{g}$ as previously reported. ${ }^{22,50}$ In the current study, we also used 5, 10, or $20 \mu \mathrm{g}$ dose of OXT to find the optimal dose for intranasal administration in CCS condition, and we found that intranasal administration of OXT $10 \mu \mathrm{g}$ or $20 \mu \mathrm{g}$ significantly decreased the CRF mRNA expression and the corticosterone level, but not with $5 \mu \mathrm{g}$. However, intranasal administration of OXT $20 \mu \mathrm{g}$ significantly increased the oxytocin mRNA expression and the serum OXT level, but not with $5 \mu \mathrm{g}$ and $10 \mu \mathrm{g}$. A previous study also showed that intranasal administration of OXT $20 \mu \mathrm{g}$ significantly increased both central and peripheral OXT concentration. ${ }^{22}$

Our present study found that high dose of OXT (20 $\mu \mathrm{g})$ significantly antagonized the inhibitory effects of CCS on GE and FPO. However, gastric phase III-like contractions and distal colonic contraction were partially restored at intranasal administration of OXT $10 \mu \mathrm{g}$ and $20 \mu \mathrm{g}$. Our current results provide a good supplement to the doses of using OXT in the intranasal administration approach.

As mentioned above, it seems that only high-concentration OXT works in the CCS rat model, which indicated that in the higher intensity stress conditions, when OXT system is exhausted, external administration of high-concentration OXT was expected in the termination of the stress response of CCS condition. Further, we cannot completely exclude that a certain amount of OXT was absorbed into the systemic circulation via blood capillaries of the nasal cavity and that, as a consequence, some peripheral effects of OXT might be the reason for some of the observed experimental results, and further investigation is needed to illustrate this issue.

In summary, our present study found that in CCS conditions, an increased $C R F$ mRNA expression and attenuated oxytocin mRNA expression at the PVN was observed. The increased CRF mRNA expression in response to CCS was significantly antagonized by intranasal administration of OXT. These results suggest that the effective pathway of intranasal administration of OXT in suppressing CRF expression is preserved following CCS loading. OXT is only effective under stressful conditions. Our study may contribute to the better understanding of the mechanism of stressinduced changes in GI dysmotility. Intranasal administration of OXT may be a new approach for treatment of stress-induced GI motility disorders.

Financial support: These studies were supported by the Natural Science Foundation of Liaoning Province of China (Grant No. 2013021084 to J.Z.) and the Scientific Research Foundation for the Returned Overseas Chinese Scholars, State Education Ministry (Grant No. 2013693 to J.Z.).

\section{Conflicts of interest: None.}

Author contributions: The authors have made the following declarations about their contributions: Yu Yang, Haijie Yu, Reji Babygirija, and Bei Shi performed the experiment; Weinan Sun and Xiaojiao Zheng were involved in the study supervision and critical revision of the manuscript; and Jun Zheng designed the experiment, analyzed the data, and wrote the paper.

\section{References}

1. Levy RL, Olden KW, Naliboff BD, et al. Psychosocial aspects of the functional gastrointestinal disorders. Gastroenterology 2006;130:14471458.

2. Talley NJ, Holtmann G, Walker MM. Therapeutic strategies for functional dyspepsia and irritable bowel syndrome based on pathophysiology. J Gastroenterol 2015;50:601-613.

3. Lenz HJ, Raedler A, Greten H, Vale WW, Rivier JE. Stress-induced gastrointestinal secretory and motor responses in rats are mediated by endogenous corticotropin-releasing factor. Gastroenterology 1988;95:15101517.

4. Taché Y, Martinez V, Wang L, Million M. $\mathrm{CRF}_{1}$ receptor signaling pathways are involved in stress-related alterations of colonic function and viscerosensitivity: implications for irritable bowel syndrome. Br J Pharmacol 2004;141:1321-1330.

5. Taché Y, Bonaz B. Corticotropin-releasing factor receptors and stressrelated alterations of gut motor function. J Clin Invest 2007;117:33-40. 
6. Nakade Y, Tsuchida D, Fukuda H, Iwa M, Pappas TN, Takahashi T. Restraint stress delays solid gastric emptying via a central CRF and peripheral sympathetic neuron in rats. Am J Physiol Regul Integr Comp Physiol 2005;288:R427-R432.

7. Nakade Y, Fukuda H, Iwa M, et al. Restraint stress stimulates colonic motility via central corticotropin-releasing factor and peripheral $5-\mathrm{HT}_{3}$ receptors in conscious rats. Am J Physiol Gastrointest Liver Physiol 2007;292:G1037-G1044.

8. Zheng J, Babygirija R, Bülbül M, Cerjak D, Ludwig K, Takahashi T. Hypothalamic oxytocin mediates adaptation mechanism against chronic stress in rats. Am J Physiol Gastrointest Liver Physiol 2010;299:G946G953.

9. Choudhury BK, Shi XZ, Sarna SK. Norepinephrine mediates the transcriptional effects of heterotypic chronic stress on colonic motor function. Am J Physiol Gastrointest Liver Physiol 2009;296:G1238-G1247.

10. Maillot C, Million M, Wei JY, Gauthier A, Taché Y. Peripheral corticotropin-releasing factor and stress-stimulated colonic motor activity involve type 1 receptor in rats. Gastroenterology 2000;119:1569-1579.

11. Kormos V, Gaszner B. Role of neuropeptides in anxiety, stress, and depression: from animals to humans. Neuropeptides 2013;47:401-419.

12. Bali A, Singh N, Jaggi AS. Neuropeptides as therapeutic targets to combat stress-associated behavioral and neuroendocrinological effects. CNS Neurol Disord Drug Targets 2014;13:347-368.

13. Liu S, Chang J, Long $\mathrm{N}$, et al. Endogenous CRF in rat large intestine mediates motor and secretory responses to stress. Neurogastroenterol Motil 2016;28:281-291.

14. Windle RJ, Kershaw YM, Shanks N, Wood SA, Lightman SL, Ingram CD. Oxytocin attenuates stress-induced $c$-fos mRNA expression in specific forebrain regions associated with modulation of hypothalamopituitary-adrenal activity. J Neurosci 2004;24:2974-2982.

15. Neumann ID. Involvement of the brain oxytocin system in stress coping: interactions with the hypothalamo-pituitary-adrenal axis. Prog Brain Res 2002;139:147-162.

16. Guastella AJ, Howard AL, Dadds MR, Mitchell P, Carson DS. A randomized controlled trial of intranasal oxytocin as an adjunct to exposure therapy for social anxiety disorder. Psychoneuroendocrinology 2009;34:917-923.

17. Koch SB, van Zuiden M, Nawijn L, Frijling JL, Veltman DJ, Olff M. Intranasal oxytocin as strategy for medication-enhanced psychotherapy of PTSD: salience processing and fear inhibition processes. Psychoneuroendocrinology 2014;40:242-256.

18. Koch SB, van Zuiden M, Nawijn L, Frijling JL, Veltman DJ, Olff M. Intranasal oxytocin administration dampens amygdala reactivity towards emotional faces in male and female PTSD patients. Neuropsychopharmacology 2016;41:1495-1504.

19. Veening JG, Olivier B. Intranasal administration of oxytocin: behavioral and clinical effects, a review. Neurosci Biobehav Rev 2013;37:1445-1465.

20. Babygirija R, Zheng J, Ludwig K, Takahashi T. Central oxytocin is involved in restoring impaired gastric motility following chronic repeated stress in mice. Am J Physiol Regul Integr Comp Physiol 2010;298:R157-R165.

21. Serova LI, Tillinger A, Alaluf LG, Laukova M, Keegan K, Sabban
EL. Single intranasal neuropeptide $\mathrm{Y}$ infusion attenuates development of PTSD-like symptoms to traumatic stress in rats. Neuroscience 2013;236:298-312.

22. Neumann ID, Maloumby R, Beiderbeck DI, Lukas M, Landgraf R. Increased brain and plasma oxytocin after nasal and peripheral administration in rats and mice. Psychoneuroendocrinology 2013;38:1985-1993.

23. Calcagnoli F, Kreutzmann JC, de Boer SF, Althaus M, Koolhaas JM. Acute and repeated intranasal oxytocin administration exerts anti-aggressive and pro-affiliative effects in male rats. Psychoneuroendocrinology 2015;51:112-121.

24. Yang Y, Babygirija R, Zheng J, et al. Central neuropeptide Y plays an important role in mediating the adaptation mechanism against chronic stress in male rats. Endocrinology 2018;159:1525-1536.

25. Yang X, Xi TF, Li YX, et al. Oxytocin decreases colonic motility of cold water stressed rats via oxytocin receptors. World J Gastroenterol 2014;20:10886-10894.

26. Paxinos G, Watson C. The rat brain in stereotaxic coordinates. 3rd ed. San Francisco: Academic 1997.

27. Zheng J, Dobner A, Babygirija R, Ludwig K, Takahashi T. Effects of repeated restraint stress on gastric motility in rats. Am J Physiol Regul Integr Comp Physiol 2009;296:R1358-R1365.

28. Lenz HJ, Burlage M, Raedler A, Greten H. Central nervous system effects of corticotropin-releasing factor on gastrointestinal transit in the rat. Gastroenterology 1988;94:598-602.

29. Lin MJ, Yu BP. Role of high-affinity choline transporter 1 in colonic hypermotility in a rat model of irritable bowel syndrome. J Neurogastroenterol Motil 2018;24:643-655.

30. Gourcerol G, Wu SV, Yuan PQ, et al. Activation of corticotropin-releasing factor receptor 2 mediates the colonic motor coping response to acute stress in rodents. Gastroenterology 2011;140:1586-1596.

31. Yuan PQ, Million M, Wu SV, Rivier J, Taché Y. Peripheral corticotropin releasing factor $(\mathrm{CRF})$ and a novel $\mathrm{CRF}_{1}$ receptor agonist, stressin1-A activate $\mathrm{CRF}_{1}$ receptor expressing cholinergic and nitrergic myenteric neurons selectively in the colon of conscious rats. Neurogastroenterol Motil 2007;19:923-936.

32. Takahashi T. Mechanism of interdigestive migrating motor complex. J Neurogastroenterol Motil 2012;18:246-257.

33. Ariga H, Imai K, Chen C, Mantyh C, Pappas TN, Takahashi T. Fixed feeding potentiates interdigestive gastric motor activity in rats: importance of eating habits for maintaining interdigestive MMC. Am J Physiol Gastrointest Liver Physiol 2008;294:G655-G659.

34. Neumann ID. Brain oxytocin: a key regulator of emotional and social behaviours in both females and males. J Neuroendocrinol 2008;20:858865 .

35. Kosfeld M, Heinrichs M, Zak PJ, Fischbacher U, Fehr E. Oxytocin increases trust in humans. Nature 2005;435:673-676.

36. Wynn JK, Green MF, Hellemann G, Reavis EA, Marder SR. A dosefinding study of oxytocin using neurophysiological measures of social processing. Neuropsychopharmacology 2019;44:289-294.

37. Kruppa JA, Gossen A, Oberwelland Wei $\beta$ E, et al. Neural modulation of social reinforcement learning by intranasal oxytocin in male adults with high-functioning autism spectrum disorder: a randomized trial. Neuro- 
psychopharmacology 2019;44:749-756.

38. Neumann ID, Krömer SA, Toschi N, Ebner K. Brain oxytocin inhibits the (re)activity of the hypothalamo-pituitary-adrenal axis in male rats: involvement of hypothalamic and limbic brain regions. Regul Pept 2000;96:31-38.

39. Ebner K, Bosch OJ, Krömer SA, Singewald N, Neumann ID. Release of oxytocin in the rat central amygdala modulates stress-coping behavior and the release of excitatory amino acids. Neuropsychopharmacology 2005;30:223-230.

40. Babygirija R, Bülbül M, Yoshimoto S, Ludwig K, Takahashi T. Central and peripheral release of oxytocin following chronic homotypic stress in rats. Auton Neurosci 2012;167:56-60.

41. Bülbül M, Babygirija R, Ludwig K, Takahashi T. Central oxytocin attenuates augmented gastric postprandial motility induced by restraint stress in rats. Neurosci Lett 2010;479:302-306.

42. Inenaga K, Yamashita H. Excitation of neurones in the rat paraventricular nucleus in vitro by vasopressin and oxytocin. J Physiol 1986;370:165180 .

43. Bülbül M, Babygirija R, Cerjak D, Yoshimoto S, Ludwig K, Takahashi T. Hypothalamic oxytocin attenuates CRF expression via GABAA receptors in rats. Brain Res 2011;1387:39-45.
44. Cullinan WE, Ziegler DR, Herman JP. Functional role of local GABAergic influences on the HPA axis. Brain Struct Funct 2008;213:63-72.

45. Di S, Tasker JG. Rapid synapse-specific regulation of hypothalamic magnocellular neurons by glucocorticoids. Prog Brain Res 2008;170:379388.

46. Huber D, Veinante P, Stoop R. Vasopressin and oxytocin excite distinct neuronal populations in the central amygdala. Science 2005;308:245-248.

47. Welch MG, Anwar M, Chang CY, et al. Combined administration of secretin and oxytocin inhibits chronic colitis and associated activation of forebrain neurons. Neurogastroenterol Motil 2010;22:654-e202.

48. Qin J, Feng M, Wang C, Ye Y, Wang PS, Liu C. Oxytocin receptor expressed on the smooth muscle mediates the excitatory effect of oxytocin on gastric motility in rats. Neurogastroenterol Motil 2009;21:430-438.

49. Welch MG, Tamir H, Gross KJ, Chen J, Anwar M, Gershon MD. Expression and developmental regulation of oxytocin (OT) and oxytocin receptors (OTR) in the enteric nervous system (ENS) and intestinal epithelium. J Comp Neurol 2009;512:256-270.

50. Born J, Lange T, Kern W, McGregor GP, Bickel U, Fehm HL. Sniffing neuropeptides: a transnasal approach to the human brain. Nat Neurosci 2002;5:514-516. 